Article

\title{
Classification of Efficient Total Domination Sets of Circulant Graphs of Degree 5
}

\author{
Young Soo Kwon ${ }^{1}$ and Moo Young Sohn ${ }^{2, *}$ \\ 1 Department of Mathematics, Yeungnam University, Kyongsan 712-749, Korea; ysookwon@ynu.ac.kr \\ 2 Department of Mathematics, Changwon National University, Changwon 641-773, Korea \\ * Correspondence: mysohn@changwon.ac.kr
}

Received: 4 November 2020; Accepted: 24 November 2020; Published: 25 November 2020

\begin{abstract}
An efficient total dominating set $D$ of a graph $G$ is a vertex subset such that every vertex of $G$ has exactly one neighbor in the set $D$. In this paper, we give necessary and sufficient conditions for the existence of efficient total domination sets of circulant graphs whose degree is 5 and classify these sets.
\end{abstract}

Keywords: graphs; efficient total domination sets; circulant graphs

\section{Introduction}

All graphs considered in this paper are finite, simple, undirected, and connected. For a graph $G$, we denote the vertex set and edge set by $V(G)$ and $E(G)$, respectively. A total domination set of $G$ with no isolated vertex is a subset $D$ of $V(G)$ such that every vertex in $G$ is adjacent to a vertex in $D$. An efficient total dominating set $D$ of a graph $G$ is a total dominating set of $G$ such that every vertex of $G$ has exactly one neighbor in the set $D$. Please note that a graph $G$ has an efficient total dominating set if and only if each component of $G$ has an efficient total dominating set. So we only consider connected graphs in this paper. For a connected graph $G$ and for a vertex $u \in V(G)$, let $N(u)$ be the set of neighbors of $u$. For any positive integer $i$, let $N_{i}(u)$ be the set of vertices $v$ such that $d(u, v)=i$, where $d(u, v)$ is the distance between $u$ and $v$. Please note that for an efficient total dominating set $D$ of $G$, if $u$ is an element of $D$, then $N_{2}(u) \cap D=\varnothing$. For more information on dominating sets of graphs, we refer the readers to [1].

Let $\Gamma$ be a finite group and $X \subseteq \Gamma \backslash\left\{1_{\Gamma}\right\}$ be a generating set for $\Gamma$ which is closed under taking inverses. The Cayley graph $\mathrm{C}(\Gamma, X)$ has vertex set $\Gamma$ with any two vertices $g, h \in \Gamma$ joined by an edge whenever $g^{-1} h \in X$. It follows that the Cayley graphs considered in this paper are finite, connected and undirected. Please note that a Cayley graph $G=C(\Gamma, X)$ is very symmetric graph because the automorphism group of $G$ contains a subgroup isomorphic to $\Gamma$ which acts regularly on $V(G)$ [2]. For a positive integer $n$, if $\Gamma$ is a cyclic group $\mathbb{Z}_{n}$, the Cayley graph $C\left(\mathbb{Z}_{n}, X\right)$ is called the circulant graph and denoted by $C_{n}\left[a_{1}, \ldots, a_{k}\right]$, where $X=\left\{ \pm a_{1}, \ldots, \pm a_{k}\right\}$. For a Cayley graph $G=C(\Gamma, X), D \subset \Gamma$ is a total dominating set (an efficient total dominating set, resp.) if and only if $D \circ X=\Gamma$ as a set (a multiset, resp.) [3,4], where $D \circ X=\{g x \mid g \in D$ and $x \in X\}$. Please note that any connected graph of order at least 2 contains total dominating set but not all connected graphs contain efficient total dominating set.

Efficient total dominating sets were first studied by Gavlas, Schultz, and Slater [5]. The problem of deciding whether a graph has an efficient total dominating set was shown to be NP-complete in general by Bakker and van Leeuwen [6] (also see [5]), and even when restricted to planar bipartite graphs of maximum degree three by Schaudt [7]. Lee proved that a Cayley graph on an Abelian group has an efficient dominating set if and only if it is a covering graph of a complete graph [8]. MacGillivray and 
Warren showed that the same methods can be used to relate efficient total dominating sets in Cayley graphs to covers of a reflexive complete graph [9]. Obradović et al. characterized the circulant graphs of degree two, three and four that have efficient dominating sets [10]. A consequence of their results is that, for circulant graphs of degree two, three or four, the vertices in the efficient dominating sets are equally spaced, i.e., are a coset of a subgroup of $\mathbb{Z}_{n}$. In [11], the authors and Chen classified all circulant graphs of degree 4 containing an efficient total dominating set. In [12], Feng et al. obtained a necessary and sufficient condition for the existence of efficient total dominating set of a circulant graph whose degree is an odd prime $p$.

The present paper deals with classification of efficient total domination sets of circulant graphs $C_{n}\left[a, b, \frac{n}{2}\right]$ whose degree is 5 . The paper is organized as follows. In Section 2, we give some properties of circulant graphs and efficient total domination sets of circulant graphs. In Sections 3, we construct efficient total domination sets of circulant graphs $C_{10 m}\left[a, b, \frac{n}{2}\right]$ with some conditions for $a$ and $b$, where $m$ is a positive integer. In Section 4, we classify all efficient total dominating sets of circulant graphs of degree 5.

\section{Preliminaries}

For a circulant graph $G=C_{n}\left[a_{1}, \ldots, a_{k}\right]$ of degree $d=2 k-1$ or $2 k$, suppose that $G$ has an efficient total dominating set $D$. Then for any $g \in D$, there uniquely exists $g^{\prime} \in D \backslash\{g\}$ adjacent to $g$. For any such elements $g$ and $g^{\prime}$ in $D$, the set $N\left(\left\{g, g^{\prime}\right\}\right)=N(g) \cup N\left(g^{\prime}\right)$ is composed of $2 d$ vertices. Furthermore, the vertex set of $G$ is a disjoint union of such sets $N\left(\left\{g, g^{\prime}\right\}\right)$, and hence $2 d$ should divide $n$, namely $n=2 d m$ for some positive integer $m$. So it suffices to consider circulant graphs of order $2 d m$ for classifying circulant graphs of degree $d$ which contain an efficient total dominating set.

A connected circulant graph $C_{n}[a]$ of degree 2 is a cycle and $C_{n}[a]$ has an efficient total domination set if and only if $n$ is a multiple of 4 . A connected circulant graph $C_{n}\left[a, \frac{n}{2}\right]$ of degree 3 is a Möbius ladder and one can show that $C_{n}\left[a, \frac{n}{2}\right]$ has an efficient total domination set if and only if $n$ is a multiple of 6 . For a circulant graph $C_{n}[a, b]$ of degree 4 , the authors and Chen [11] obtained the following result.

Theorem 1 ([11]). Let $G=C_{8 m}[a, b]$ be a connected circulant graph of degree 4 with $a \equiv 1(\bmod 8)$ and $b \equiv$ $0,1,2,3$ or $4(\bmod 8)$. Now $G$ has an efficient total dominating set if and only if $b \equiv 3(\bmod 8)$ or $(b \equiv 1(\bmod 8)$ and $\operatorname{gcd}(8 m,|a-b|)=\operatorname{gcd}(4 m,|a-b|))$.

Please note that any connected circulant graph $C_{8 m}[a, b]$ of degree 4 is isomorphic to a circulant graph $C_{8 m}[c, d]$ such that $c \equiv 1(\bmod 8)$ and $d \equiv 0,1,2,3$ or $4(\bmod 8)$.

For a connected circulant graph $G=C_{10 m}[a, b, 5 m]$ of degree 5 , assume that $G$ has an efficient total dominating set $D$. If for any $x \in D, x+5 m$ also belongs to $D$, then we call $D$ a type-I efficient total dominating set of $G$. If there exist $x, y \in D$ such that $x+5 m \in D$ and $y+5 m \notin D$, then we call $D$ a type-II efficient total dominating set of $G$. If there is no $x \in D$ such that $x+5 m \in D$, then $D$ is called a type-III efficient total dominating set of $G$. The following is the main theorem in our paper.

Theorem 2. Let $G=C_{10 m}[a, b, 5 m]$ be a connected circulant graph of degree 5 . Now we have

(1) G has a type-I efficient total dominating set if and only if $\{ \pm a, \pm b\} \equiv\{1,2,3,4\}$ ( $\bmod 5)$. In this case, any type-I efficient total dominating set of $G$ is the subgroup $\langle 5\rangle$ of $\mathbb{Z}_{10 m}$ or its coset.

(2) There is no type-II efficient total dominating set of G.

(3) G has a type-III efficient total dominating set if and only if $m$ is odd and $\{ \pm a, \pm b\} \equiv\{1,3,7,9\}(\bmod 10)$. In this case, any type-III efficient total dominating set of $G$ is $(\langle 10\rangle+i) \cup(\langle 10\rangle+j)$, where $i$ is even and $j$ is odd with $j \neq i+5(\bmod 10)$. 


\section{Constructions}

For $G_{1}=C_{10 m}[a, b, 5 m]$ with $\{ \pm a, \pm b\} \equiv\{1,2,3,4\}(\bmod 5)$, let

$$
D_{1}=\left\{x \in \mathbb{Z}_{10 m} \mid x \equiv 0(\bmod 5)\right\}=\langle 5\rangle .
$$

Please note that $\{ \pm a, \pm b, 5 m\} \equiv\{0,1,2,3,4\}(\bmod 5)$. So for any $y \in \mathbb{Z}_{10 m}$, we have $\mid\{y+a, y-$ $a, y+b, y-b, y+5 m\} \cap D_{1} \mid=1$, namely $D_{1}$ is an efficient total dominating set of $G_{1}$. In this case, for any $x \in D_{1}, x+5 m$ is also an element of $D_{1}$, and hence $D_{1}$ is a type-I efficient total dominating set of $G_{1}$.

Let $G_{2}=C_{10 m}[a, b, 5 m]$ satisfy that $m$ is odd and $\{ \pm a, \pm b\} \equiv\{1,3,7,9\}(\bmod 10)$. For any $i=1,3,7,9$, let

$$
D_{2}(i)=\left\{x \in \mathbb{Z}_{10 m} \mid x \equiv 0 \text { or } i(\bmod 10)\right\}=\langle 10\rangle \cup(\langle 10\rangle+i) .
$$

Please note that $\{ \pm a, \pm b, 5 m\} \equiv\{1,3,5,7,9\}(\bmod 10)$ because $m$ is odd. So for any $y \in \mathbb{Z}_{10 m}$, we have $\left|\{y+a, y-a, y+b, y-b, y+5 m\} \cap D_{2}(i)\right|=1$, and hence $D_{2}(i)$ is an efficient total dominating set of $G_{2}$. In this case, for any $x \in D_{2}(i), x+5 m$ is not an element of $D_{2}(i)$ because $x+5 m \equiv 5$ or $i+5(\bmod 10)$. Therefore $D_{2}(i)$ is a type-III efficient total dominating set of $G_{2}$.

In the next section, we prove Theorem 2 by showing that these efficient total dominating sets are all such sets up to translation.

\section{Proof of Main Theorem}

For a circulant graph $G=C_{n}\left[a_{1}, \ldots, a_{k}\right]$ of degree $d$, if $n$ is not a multiple of $2 d$, then there is no efficient total dominating set of $G$. So to prove Theorem 2, it suffices to consider a circulant graph $G=C_{10 m}[a, b, 5 \mathrm{~m}]$ of degree 5. Please note that if $D$ is an efficient total dominating set of $G=C_{10 m}[a, b, 5 m]$, then for any $s \in \mathbb{Z}_{10 m}, s+D=\{s+x \mid x \in D\}$ is also an efficient total dominating set of $G$. So from now on, we assume that 0 is an element of any efficient total dominating set of $G$.

For the first case, we aim to consider type-I efficient total dominating set of $G$. For classifying type-I efficient total dominating sets, we need the following proposition.

Proposition 1 ([10]). For a connected circulant graph $G=C_{n}[a, b]$ of degree $4, G$ has an efficient dominating set if and only if $n$ is a multiple of 5 and $\{ \pm a, \pm b\} \equiv\{1,2,3,4\}(\bmod 5)$.

Remark 1. Let $G=C_{n}[a, b]$ be a connected circulant graph of degree 4 and satisfy that $n$ is a multiple of 5 and $\{ \pm a, \pm b\} \equiv\{1,2,3,4\}(\bmod 5)$. In the proof of Proposition 1 in [10], it was showed that the subgroup $\langle 5\rangle$ of $\mathbb{Z}_{n}$ is the unique efficient dominating set containing 0 .

Assume that there exists a type-I efficient total dominating set $D_{1}$ of $G=C_{10 m}[a, b, 5 m]$. For any $k \in \mathbb{Z}_{10 m}$, let $\bar{k}$ be an element of $\mathbb{Z}_{5 m}$ such that $\bar{k} \equiv k(\bmod 5 m)$. Please note that $\overline{5 m}=0$ in $\mathbb{Z}_{5 m}$. For our convenience, let $\bar{G}=C_{5 m}[\bar{a}, \bar{b}]$ and $\bar{D}_{1}=\left\{\bar{x} \mid x \in D_{1}\right\}$ as a subset of $\mathbb{Z}_{5 m}$. Now we have the following lemma.

Lemma 1. For a connected circulant graph $G=C_{10 m}[a, b, 5 \mathrm{~m}]$ of degree 5 , if there exists a type-I efficient total dominating set $D_{1}$ of $G$, then the circulant graph $\bar{G}=C_{5 m}[\bar{a}, \bar{b}]$ is simple and connected, and $\bar{D}_{1}$ is an efficient dominating set of $\bar{G}$.

Proof of Lemma 1. If $\bar{a}$ equals to $\bar{b}$ or $-\bar{b}$ as elements of $\mathbb{Z}_{5 m}$, then $a=b+5 m$ or $a=-b+5 m$, and hence $a$ is adjacent to both 0 and $5 m$ in $G$. Since both 0 and $5 m$ are elements of $D_{1}$, it contradicts the assumption that $D_{1}$ is an efficient total dominating set of $G$. So $\bar{G}$ is simple. 
Since $G$ is connected, for any $y \in \mathbb{Z}_{10 m}$, there exists a path $0, x_{1}, x_{1}+x_{2}, \ldots, x_{1}+x_{2}+\cdots+x_{k}=y$, where for any $i=1, \ldots, k, x_{i} \in\{ \pm a, \pm b, 5 m\}$. This implies 0 and $\bar{y}$ belong to the same component of $\bar{G}$, and hence $\bar{G}$ is connected.

For any $z \in \mathbb{Z}_{10 m} \backslash D_{1}$, we have $\left|\{z+a, z-a, z+b, z-b\} \cap D_{1}\right|=1$ because $D_{1}$ is a type-I efficient total dominating set of $G$. This implies that for any $\bar{z} \in \mathbb{Z}_{5 m} \backslash \bar{D}_{1},\left|\{\bar{z}+\bar{a}, \bar{z}-\bar{a}, \bar{z}+\bar{b}, \bar{z}-\bar{b}\} \cap \bar{D}_{1}\right|=1$. Therefore $\bar{D}_{1}$ is an efficient dominating set of $\bar{G}$.

Using Proposition 1 and Lemma 1, one can prove Theorem 2(1) as follows:

Proof of Theorem 2(1). By a construction in Section 3, we know that if $\{ \pm a, \pm b\} \equiv\{1,2,3,4\}(\bmod 5)$, then $G=C_{10 m}[a, b, 5 m]$ has a type-I efficient total dominating set.

Conversely, assume that $G$ has a type-I efficient total dominating set $D_{1}$ containing 0 . By Lemma 1 , $\bar{D}_{1}$ is an efficient dominating set of $\bar{G}=C_{5 m}[\bar{a}, \bar{b}]$. Hence we have $\{ \pm \bar{a}, \pm \bar{b}\} \equiv\{1,2,3,4\}(\bmod 5)$ by Proposition 1. This implies that $\{ \pm a, \pm b\} \equiv\{1,2,3,4\}(\bmod 5)$. Furthermore, $\bar{D}_{1}$ is the subgroup $\langle 5\rangle$ of $\mathbb{Z}_{5 m}$ (see Remark 1 ). This means that $D_{1}$ is the subgroup $\langle 5\rangle$ of $\mathbb{Z}_{10 m}$.

From now on, we aim to consider a type-II or type-III efficient total dominating set of $G=C_{10 m}[a, b, 5 m]$.

Lemma 2. Let $D$ be an efficient total dominating set of $G=C_{10 m}[a, b, 5 m]$. For any $x \in D$ and for any $\alpha, \beta$ satisfying $\{ \pm \alpha, \pm \beta\}=\{ \pm a, \pm b\}$, we have

(1) $\quad N_{2}(x) \cap D=\varnothing$,

(2) $x+2 \alpha+2 \beta \notin D$,

(3) $x+3 \alpha+5 m \notin D$, and

(4) $\quad x+4 \alpha \notin D$.

Proof of Lemma 2. (1) Suppose that $N_{2}(x) \cap D \neq \varnothing$ and let $y \in N_{2}(x) \cap D$. Then there exists a common neighbor $z$ of $x$ and $y$, and hence $z$ is dominated by $x$ and $y$, a contradiction.

(2) Suppose that $x+2 \alpha+2 \beta \in D$. Then, $N(x+\alpha+\beta+5 m) \backslash\left(N_{2}(x) \cup N_{2}(x+2 \alpha+2 \beta)\right)=\varnothing$, and hence there is no element in $D$ which dominates $x+\alpha+\beta+5 m$, a contradiction.

(3) Suppose that $x+3 \alpha+5 m \in D$. Then, $N(x+2 \alpha+\beta) \backslash\left(N_{2}(x) \cup N_{2}(x+3 \alpha+5 m)\right)=\{x+2 \alpha+2 \beta\}$, and hence $x+2 \alpha+2 \beta$ should be an element of $D$ to dominate $x+2 \alpha+\beta$. It contradicts (2).

(4) Suppose that $x+4 \alpha \in D$. Since $N(x+2 \alpha+5 m) \backslash\left(N_{2}(x) \cup N_{2}(x+4 \alpha)\right)=\{x+2 \alpha+\beta+5 m, x+2 \alpha-$ $\beta+5 m\}$, we have $x+2 \alpha+\beta+5 m \in D$ or $x+2 \alpha-\beta+5 m \in D$. Without loss of generality, assume that $x+2 \alpha+\beta+5 m \in D$. Now $N(x+\alpha-\beta+5 m) \backslash\left(N_{2}(x) \cup N_{2}(x+2 \alpha+\beta+5 m)\right)=\{x+\alpha-2 \beta+5 m\}$, and hence $x+\alpha-2 \beta+5 m \in D$. In this situation, $N(x+2 \alpha-\beta) \backslash\left(N_{2}(x+\alpha-2 \beta+5 m) \cup N_{2}(x+4 \alpha)\right)=\varnothing$. Therefore, there is no element in $D$ dominating $x+2 \alpha-\beta$, a contradiction.

Corollary 1. Let $D$ be an efficient total dominating set of $G=C_{10 m}[a, b, 5 m]$. If $x \in D$, then we have

(2) $\quad\{x \pm 3 a+5 m, x \pm 3 b+5 m\} \cap D=\varnothing$ and

(3) $\quad\{x \pm 4 a, x \pm 4 b\} \cap D=\varnothing$.

Proof of Corollary 1. (1) By Lemma 2(2), we have $\{x \pm 2 a \pm 2 b\} \cap D=\varnothing$.

(2) By Lemma 2(3), we have $\{x \pm 3 a+5 m, x \pm 3 b+5 m\} \cap D=\varnothing$.

(3) By Lemma 2(4), we have $\{x \pm 4 a, x \pm 4 b\} \cap D=\varnothing$. 
Lemma 3. Let $G=C_{10 m}[a, b, 5 \mathrm{~m}]$ be a connected circulant graph of degree 5. If there is a cycle of length 3 in $G$, then there is neither a type-II efficient total dominating set nor a type-III efficient total dominating set in $G$.

Proof of Lemma 3. Assume that $G$ contains a cycle $C$ of length 3 and let $V(C)=\{0, x, y\}$. Suppose that there exists a type-II or type-III efficient total dominating set $D$ of $G$ which contains 0 . Note that $D$ contains at most one vertex in any cycle of length 3 . So $D$ contains neither $x$ nor $y$.

For the first case, suppose that $C$ contain $5 \mathrm{~m}$. Without loss of generality, assume that $x=5 \mathrm{~m}$ and $y=a$. Then $a+5 m \in\{-a, \pm b\}$. If $a+5 m=b$ or $-b$, then for any $z \in\{ \pm a, \pm b, 5 m\}$, there exists a cycle of length 3 containing both 0 and $z$. This implies that $z \notin D$ and hence 0 cannot be dominated by $D$, a contradiction. So we may assume that $a+5 m=-a$, namely $2 a=5 m$. Now the subgraph induced by $\{0, a,-a, 5 m\}$ is a complete graph $K_{4}$. For $D$ to dominate 0 , we have $b \in D$ or $-b \in D$. Without loss of generality, let $b \in D$. Now $\{-b+a,-b-a,-b+5 m\}$ is a subset of $N_{2}(0)$ and hence $\{-b+a,-b-a,-b+5 m\} \cap D=\varnothing$. Since neither $a$ nor $-b$ is an element of $D$ and $a+5 m=-a,-2 b+a$ should be an element of $D$ to dominate $-b+a$. By a similar reason, $-2 b-a$ should be an element of $D$ to dominate $-b-a$. Now $-2 b$ is dominated by both $-2 b+a$ and $-2 b-a$, a contradiction.

Therefore, we may assume that there is no cycle of length 3 containing both 0 and $5 m$, and hence $x, y \in\{ \pm a, \pm b\}$. Since $C$ is a cycle of length 3 containing $0, x$ and $y$, we have $x, y-x,-y \in\{ \pm a, \pm b\}$ and at least two of $x, y-x,-y$ are the same element. Without loss of generality, assume that $x=y-x=a$, namely $x=a$ and $y=2 a$. Now, we can divide two cases as $2 a=-a$ and $(2 a=b$ or $2 a=-b)$.

First assume that $2 a=-a$. Since 0 is an element of $D$, neither $a$ nor $-a$ is an element of $D$, and hence exactly one of $5 m, b$ and $-b$ is an element of $D$. If $5 m \in D$, then $\{b, b \pm a, b+5 m, b \pm a+5 m\}$ is a subset of $N_{2}(0) \cup N_{2}(5 m)$. So $\{b, b \pm a, b+5 m, b \pm a+5 m\} \cap D=\varnothing$. To dominates $b \pm a, 2 b \pm a$ should be elements of $D$. Now $2 b$ is dominated by both $2 b+a$ and $2 b-a$, a contradiction. For the remaining case, assume that $b \in D$ or $-b \in D$. Without loss of generality, let $b \in D$. Since $\{ \pm a, \pm a+5 m, \pm a+b+5 m\} \subset N_{2}(0) \cup N_{2}(b)$, $\pm a-b+5 m$ should be elements of $D$ to dominate $\pm a+5 m$. Now $-b+5 m$ is dominated by both $a-b+5 m$ and $-a-b+5 m$, a contradiction.

For the last case, assume that $2 a=b$ or $2 a=-b$. Then for any $k \in \mathbb{Z}_{10 m}$ and for any $\alpha \in\{ \pm a, \pm b\}$, there exists a cycle of length 3 containing both $k$ and $k+\alpha$. Hence if $k \in D$, then $k+5 m$ is an element of $D$. This implies that $D$ is a type-I efficient total dominating set of $G$, a contradiction.

Please note that there possibly exists a type-I efficient total dominating set of $G=C_{10 \mathrm{~m}}[a, b, 5 \mathrm{~m}]$ even though there is a cycle of length 3 in $G$. For example, let $G=C_{20}[1,2,10]$ and $D=\{0,5,10,15\}$. Now the subgraph of $G$ induced by $\{0,1,2\}$ is a cycle of length 3 and $D$ is a type-I efficient total dominating set of $G$.

Lemma 4. Let $G=C_{10 m}[a, b, 5 m]$ be a connected circulant graph of degree 5. For a type-II or type-III efficient total dominating set $D$ of $G$, if $x, x+\alpha, x+2 \beta+5 m, x+\alpha+2 \beta+5 m \in D$ for some $\alpha, \beta$ such that $\{ \pm \alpha, \pm \beta\}=$ $\{ \pm a, \pm b\}$, then we have $x+3 \alpha+\beta, x+3 \alpha+\beta+5 m, x-2 \alpha+\beta, x-2 \alpha+\beta+5 m \in D$.

Proof of Lemma 4. Let $S=N_{2}(x) \cup N_{2}(x+\alpha) \cup N_{2}(x+2 \beta+5 m) \cup N_{2}(x+\alpha+2 \beta+5 m)$. Since $N(x+$ $2 \alpha+\beta)-S=\{x+3 \alpha+\beta\}$ and $N(x+2 \alpha+\beta+5 m)-S=\{x+3 \alpha+\beta+5 m\}$, we have $x+3 \alpha+\beta, x+$ $3 \alpha+\beta+5 m \in D$. By a similar way, one can show that $x-2 \alpha+\beta, x-2 \alpha+\beta+5 m \in D$.

Lemma 4 implies that there is no type-III efficient total dominating set $D$ of $G$ containing $x, x+\alpha, x+$ $2 \beta+5 m, x+\alpha+2 \beta+5 m$ for some $x \in \mathbb{Z}_{10 m}$ and for some $\alpha, \beta$ such that $\{ \pm \alpha, \pm \beta\}=\{ \pm a, \pm b\}$.

Now, we aim to show that there is no type-II efficient total dominating set of $G=C_{10 \mathrm{~m}}[a, b, 5 \mathrm{~m}]$. For the contrary, suppose that there exists a type-II efficient total dominating set $D_{2}$ of $G$. 
Lemma 5. Let $G=C_{10 m}[a, b, 5 m]$ be a connected circulant graph of degree 5. For a type-II efficient total dominating set $D_{2}$ of $G$, there exists $x \in D_{2}$ and $\alpha, \beta \in\{ \pm a, \pm b\}$ with $\beta \neq \pm \alpha$ such that $x+5 m \in D_{2}$ and $\left|\{x+2 \alpha+\beta, x+2 \alpha+\beta+5 m\} \cap D_{2}\right|=1$.

Proof of Lemma 5. Assume that $0,5 m \in D_{2}$. Let $y$ be an element in $D_{2}$ such that $y+5 m \notin D_{2}$ and the distance $d(0, y)$ is the smallest among such vertices. Let $0=z_{0}, z_{1}, \ldots, z_{k}=y$ be the shortest path between 0 and $y$. Please note that $k \geq 3$ and $z_{k-2}, z_{k-1} \notin D_{2}$. To dominate $z_{k-2}$, there exists $x \in N\left(z_{k-2}\right)$ such that $x \in D_{2}$. Since the distance $d(0, x)$ is shorter than the distance $d(0, y), x+5 m$ is also an element of $D_{2}$. Please note that the distance $d(y, x)$ is 3. By Lemma 2(3), $y=x+2 \alpha+\beta$ for some $\alpha, \beta$ such that $\{ \pm \alpha, \pm \beta\}=\{ \pm a, \pm b\}$.

Lemma 6. Let $G=C_{10 m}[a, b, 5 m]$ be a connected circulant graph of degree 5. For a type-II efficient total dominating set $D_{2}$ of $G$, if $x, x+5 m, x+2 \alpha+\beta \in D_{2}$ and $x+2 \alpha+\beta+5 m \notin D_{2}$ for some $\alpha, \beta$ such that $\{ \pm \alpha, \pm \beta\}=\{ \pm a, \pm b\}$, then we have

(1) all of $x+\alpha+2 \beta+5 m, x+3 \alpha+\beta, x+2 \alpha-\beta+5 m$ belong to $D_{2}$.

(2) all of $x \pm(2 \alpha+\beta), x \pm(3 \alpha+\beta), x \pm(2 \alpha-\beta)+5 m, x \pm(3 \alpha-\beta)+5 m, x \pm(\alpha-2 \beta), x \pm(\alpha-$ $3 \beta), x \pm(\alpha+2 \beta)+5 m, x \pm(\alpha+3 \beta)+5 m$ belong to $D_{2}$. Furthermore, these elements are all elements in 4$$
\bigcup\left(\left(N_{i}(x) \cup N_{i}(x+5 m)\right) \cap D_{2}\right) \backslash\{x, x+5 m\} \text {. }
$$

All of $x \pm 5 \alpha, x \pm 5 \alpha+5 m, x \pm 5 \beta, x \pm 5 \beta+5 m$ belong to $D_{2}$.

Proof of Lemma 6. (1) Since $N(x+\alpha+\beta+5 m) \backslash N_{2}(x)$ is $\{x+\alpha+2 \beta+5 m, x+2 \alpha+\beta+5 m\}$ and $x+$ $2 \alpha+\beta+5 m \notin D_{2}$, the element $x+\alpha+2 \beta+5 m$ should be an element of $D_{2}$ to dominate $x+\alpha+\beta+5 m$.

Please note that $N(x+2 \alpha+\beta) \backslash\left(N_{2}(x) \cup N_{2}(x+\alpha+2 \beta+5 m)\right)$ is $\{x+3 \alpha+\beta\}$ and hence $x+$ $3 \alpha+\beta \in D_{2}$. Similarly, we have $N(x+2 \alpha+5 m) \backslash\left(N_{2}(x) \cup N_{2}(x+3 \alpha+\beta)\right)$ is $\{x+2 \alpha-\beta+5 m\}$. So $x+2 \alpha-\beta+5 m \in D_{2}$.

(2) Since $x+2 \alpha+\beta \in D_{2}$, the element $x+2 \alpha-\beta$ is not an element of $D_{2}$. Let $D_{2}^{\prime}=D_{2}+5 \mathrm{~m}$. Now $D_{2}^{\prime}$ is also a type-II efficient total dominating set of $G$ such that $x, x+5 m, x+2 \alpha-\beta \in D_{2}^{\prime}$ and $x+2 \alpha-\beta+5 m \notin D_{2}^{\prime}$. By (1), we have $x+3 \alpha-\beta \in D_{2}^{\prime}$ and $x+\alpha-2 \beta+5 m \in D_{2}^{\prime}$. This implies that $x+3 \alpha-\beta+5 m \in D_{2}$ and $x+\alpha-2 \beta \in D_{2}$. By using a similar way continually, one can show that all of $x \pm(2 \alpha+\beta), x \pm(3 \alpha+$ $\beta), x \pm(2 \alpha-\beta)+5 m, x \pm(3 \alpha-\beta)+5 m, x \pm(\alpha-2 \beta), x \pm(\alpha-3 \beta), x \pm(\alpha+2 \beta)+5 m, x \pm(\alpha+3 \beta)+5 m$ belong to $D_{2}$. Denote the set of these elements by $S$. Now it can be easily checked that any element in

$\bigcup_{i=1}^{4}\left(N_{i}(x) \cup N_{i}(x+5 m)\right) \backslash\{x, x+5 m\}$ which does not belong to $S$ is an element of $N_{2}(z)$ for some $z \in S$. Therefore $\bigcup_{i=1}^{4}\left(\left(N_{i}(x) \cup N_{i}(x+5 m)\right) \cap D_{2}\right) \backslash\{x, x+5 m\}=S$.

(3) Since $x+2 \alpha+\beta, x+3 \alpha+\beta, x+2 \alpha-\beta+5 m, x+3 \alpha-\beta+5 m \in D_{2}$, by Lemma $4, x+5 \alpha, x+5 \alpha+m \in$ $D_{2}$. By a similar way with $S$ as a subset of $D_{2}$, one can show that all of $x \pm 5 \alpha, x \pm 5 \alpha+5 m, x \pm 5 \beta, x \pm$ $5 \beta+5 m$ belong to $D_{2}$.

Lemma 7. Let $G=C_{10 m}[a, b, 5 m]$ be a connected circulant graph of degree 5 and assume that there is a type-II efficient total dominating set $D_{2}$ such that $0,5 m, 2 \alpha+\beta \in D_{2}$ and $2 \alpha+\beta+5 m \notin D_{2}$ for some $\alpha, \beta$ such that $\{ \pm \alpha, \pm \beta\}=\{ \pm a, \pm b\}$. Then $r a+s b, r a+s b+5 m \in D_{2}$ if and only if both $r$ and s are multiples of 5 . 
Proof of Lemma 7. Let $S$ be $\bigcup_{i=1}^{4}\left(\left(N_{i}(0) \cup N_{i}(5 m)\right) \cap D_{2}\right) \backslash\{0,5 m\}$. By Lemma $6, S$ is composed of $\pm(2 \alpha+$ $\beta), \pm(3 \alpha+\beta), \pm(2 \alpha-\beta)+5 m, \pm(3 \alpha-\beta)+5 m, \pm(\alpha-2 \beta), \pm(\alpha-3 \beta), \pm(\alpha+2 \beta)+5 m, \pm(\alpha+3 \beta)+5 m$, and $\pm 5 \alpha, \pm 5 \alpha+5 m, \pm 5 \beta, \pm 5 \beta+5 m \in D_{2}$. Please note that for any $x \in S$, we have $x+5 m \notin D_{2}$. Let $D_{2}^{\prime}=D_{2}+5 \alpha+5 m$. Now $D_{2}^{\prime}$ is also a type-II efficient total dominating set of $G$ and $0,5 m, 2 \alpha+\beta \in D_{2}^{\prime}$ and $2 \alpha+\beta+5 m \notin D_{2}^{\prime}$. Let $S^{\prime}$ be $\bigcup_{i=1}^{4}\left(\left(N_{i}(0) \cup N_{i}(5 m)\right) \cap D_{2}^{\prime}\right) \backslash\{0,5 m\}$. By Lemma $6, S$, is composed of $\pm(2 \alpha+\beta), \pm(3 \alpha+\beta), \pm(2 \alpha-\beta)+5 m, \pm(3 \alpha-\beta)+5 m, \pm(\alpha-2 \beta), \pm(\alpha-3 \beta), \pm(\alpha+2 \beta)+5 m, \pm(\alpha+$ $3 \beta)+5 m$, and $\pm 5 \alpha, \pm 5 \alpha+5 m, \pm 5 \beta, \pm 5 \beta+5 m \in D_{2}^{\prime}$. This implies that $-5 \alpha \pm 5 \alpha,-5 \alpha \pm 5 \alpha+5 m,-5 \alpha \pm$ $5 \beta,-5 \alpha \pm 5 \beta+5 m \in D_{2}$. By applying Lemma 6 continually, one can show that for any integers $r$ and $s$, $5 r \alpha+5 s \beta, 5 r \alpha+5 s \beta+5 m \in D_{2}$. This means that $5 r a+5 s b, 5 r a+5 s b+5 m \in D_{2}$.

So we showed sufficient part. Note that for any $x \in \bigcup_{i=1}^{4}\left(N_{i}(5 r a+5 s b) \cup N_{i}(5 r a+5 s b+5 m)\right) \cap D_{2}$ which is neither $5 r a+5 s b$ nor $5 r a+5 s b+5 m$, it holds that $x+5 m \notin D_{2}$.

Suppose that there exist integers $r$ and s such that $r a+s b, r a+s b+5 m \in D_{2}$ and at least one of $r$ and $s$ is not a multiple of 5 . Then there are integers $r_{1}$ and $s_{1}$ such that $r a+s b \notin\left\{5 r_{1} a+5 s_{1} b, 5 r_{1} a+5 s_{1} b+5 m\right\}$ and $r a+s b$ belongs to $\bigcup_{i=1}^{4}\left(N_{i}\left(5 r_{1} a+5 s_{1} b\right) \cup N_{i}\left(5 r_{1} a+5 s_{1} b+5 m\right)\right) \cap D_{2}$. This implies that $r a+s b+5 m \notin$ $D_{2}$, a contradiction.

Proof of Theorem 2(2). For a connected circulant graph $G=C_{10 m}[a, b, 5 m]$ of degree 5 , suppose that there exists a type-II efficient total dominating set $D_{2}$. By Lemma 5, there exists $x \in D_{2}$ and $\alpha, \beta \in\{ \pm a, \pm b\}$ with $\beta \neq \pm \alpha$ such that $x+5 m$ and $\left|\{x+2 \alpha+\beta, x+2 \alpha+\beta+5 m\} \cap D_{2}\right|=1$. Let $D_{2}^{\prime}=D_{2}-x$. Then $D_{2}^{\prime}$ is also a type-II efficient total dominating set and it contains 0 and $5 \mathrm{~m}$. Furthermore $\left|\{2 \alpha+\beta, 2 \alpha+\beta+5 m\} \cap D_{2}^{\prime}\right|=1$. By Lemma 7, $r a+s b, r a+s b+5 m \in D_{2}^{\prime}$ if and only if both $r$ and $s$ are multiples of 5 . Since both $b a-a b=0$ and $b a-a b+5 m=5 m$ belong to $D_{2}^{\prime}$, both $b$ and $-a$ are multiples of 5 . This implies that all generators of $G$ are multiples of 5 and hence $G$ is not connected, which is a contradiction. Therefore, there is no type-II efficient total dominating set of $G$.

For the last case, let us consider type-III efficient total dominating sets of a connected circulant graph $G=C_{10 m}[a, b, 5 m]$ of degree 5 . From now on, we assume that $D_{3}$ is a type-III efficient total dominating set of $G$.

Lemma 8. For any $\alpha, \beta$ such that $\{ \pm \alpha, \pm \beta\}=\{ \pm a, \pm b\}$, if $x, x+\alpha \in D_{3}$, then $x+2 \alpha+\beta+5 m \in D_{3}$ or $x+2 \alpha-\beta+5 m \in D_{3}$

Proof of Lemma 8. Assume that $x, x+\alpha \in D_{3}$. Then $x+2 \alpha, x+2 \alpha+5 m \notin D_{3}$. Now $N(x+2 \alpha+$ $5 m) \backslash N_{2}(x)=\{x+2 \alpha+\beta+5 m, x+2 \alpha-\beta+5 m, x+3 \alpha+5 m\}$. By Lemma 2(3), $x+3 \alpha+5 m \notin D_{3}$. So $x+2 \alpha+\beta+5 m \in D_{3}$ or $x+2 \alpha-\beta+5 m \in D_{3}$.

Lemma 9. For any $\alpha, \beta$ such that $\{ \pm \alpha, \pm \beta\}=\{ \pm a, \pm b\}$, there is no $x \in D_{3}$ such that $x+\alpha, x+2 \alpha+\beta+$ $5 m, x+2 \alpha+2 \beta+5 m \in D_{3}$.

Proof of Lemma 9. Suppose that $x, x+\alpha, x+2 \alpha+\beta+5 m, x+2 \alpha+2 \beta+5 m \in D_{3}$. Since $N(x+\beta+5 m) \backslash$ $\left(N_{2}(x+\alpha) \cup N_{2}(x+2 \alpha+2 \beta+5 m)\right)=\{x-\alpha+\beta+5 m\}$ and $N(x+\alpha-\beta+5 m) \backslash\left(N_{2}(x) \cup N_{2}(x+2 \alpha+\right.$ $\beta+5 m)=\{x+\alpha-2 \beta+5 m\}$, both $x-\alpha+\beta+5 m$ and $x+\alpha-2 \beta+5 m$ should be elements of $D_{3}$. Please note that $N(x-\beta+5 m) \backslash\left(N_{2}(x+\alpha) \cup N_{2}(x-\alpha+\beta+5 m)=\{x-2 \beta+5 m\}\right.$. This implies that $x-2 \beta+5 m \in D_{3}$. Since $x, x+\alpha, x-2 \beta+5 m, x+\alpha-2 \beta+5 m \in D_{3}$, we have $x-3 \alpha-\beta, x-3 \alpha-\beta+5 m \in$ $D_{3}$ by Lemma 4 , which contradicts the assumption that $D_{3}$ is a type-III efficient total dominating set of $G$. 
Lemma 10. For any $\alpha, \beta$ such that $\{ \pm \alpha, \pm \beta\}=\{ \pm a, \pm b\}$, if $x, x+\alpha, x+2 \alpha+\beta+5 m \in D_{3}$, then

$$
\begin{aligned}
& x+3 \alpha+\beta+5 m, x+4 \alpha+2 \beta \in D_{3}, \\
& x+\alpha-2 \beta+5 m, x+2 \alpha-2 \beta+5 m, x+3 \alpha-\beta \in D_{3} . \\
& \text { for any integers } r, s, x+r(2 \alpha+\beta)+s(\alpha-2 \beta)+5(r+s) m, x+r(2 \alpha+\beta)+s(\alpha-2 \beta)+\alpha+5(r+ \\
& \text { s) } m \in D_{3} .
\end{aligned}
$$

Proof of Lemma 10. (1) Please note that $N(x+2 \alpha+\beta+5 m) \backslash N_{2}(x+\alpha)=\{x+2 \alpha+2 \beta+5 m, x+3 \alpha+$ $\beta+5 m\}$. By Lemma 9, $x+2 \alpha+2 \beta+5 m \notin D_{3}$, and hence $x+3 \alpha+\beta+5 m \in D_{3}$. Now we have $N(x+4 \alpha+\beta) \backslash N_{2}(x+2 \alpha+\beta+5 m)=\{x+4 \alpha, x+4 \alpha+2 \beta, x+5 \alpha+\beta\}$. By Lemma 2(3) and (4), $x+5 \alpha+\beta \notin D_{3}$ and $x+4 \alpha \notin D_{3}$. Therefore $x+4 \alpha+2 \beta \in D_{3}$.

(2) Please note that $N(x+\alpha-\beta+5 m) \backslash\left(N_{2}(x) \cup N(x+2 \alpha+\beta+5 m)\right)=\{x+\alpha-2 \beta+5 m\}$. This implies that $x+\alpha-2 \beta+5 m \in D_{3}$. Now since $N(x+\alpha-2 \beta+5 m) \backslash N_{2}(x+\alpha)=\{x-2 \beta+5 m, x+\alpha-3 \beta+$ $5 m, x+2 \alpha-2 \beta+5 m\}$, exactly one of $x-2 \beta+5 m, x+\alpha-3 \beta+5 m, x+2 \alpha-2 \beta+5 m$ is an element of $D_{3}$. By Lemma 2(3), $x+\alpha-3 \beta+5 m \notin D_{3}$. Suppose that $x-2 \beta+5 m \in D_{3}$. Then by Lemma 4 , both $x+3 \alpha-\beta$ and $x+3 \alpha-\beta+5 m$ belong to $D_{3}$, which contradicts the assumption that $D_{3}$ is a type-III efficient total dominating set of $G$. So $x+2 \alpha-2 \beta+5 m \in D_{3}$.

Now since $N(x+2 \alpha-\beta) \backslash\left(N_{2}(x) \cup N_{2}(x+\alpha-2 \beta+5 m)\right)=\{x+3 \alpha-\beta\}, x+3 \alpha-\beta$ belongs to $D_{3}$. (3) By a similar way showing (1), we have $x+5 \alpha+2 \beta, x+6 \alpha+3 \beta+5 m \in D_{3}$ because $x+2 \alpha+\beta+5 m, x+$ $3 \alpha+\beta+5 m, x+4 \alpha+2 \beta \in D_{3}$. By applying similar process continually, one can show that for any integer $i$, $x+2 i(2 \alpha+\beta), x+2 i(2 \alpha+\beta)+\alpha \in D_{3}$ and $x+(2 i+1)(2 \alpha+\beta)+5 m, x+(2 i+1)(2 \alpha+\beta)+\alpha+5 m \in D_{3}$.

Since $x+\alpha-2 \beta+5 m, x+2 \alpha-2 \beta+5 m, x+3 \alpha-\beta \in D_{3}$, we get $x+2 \alpha-4 \beta, x+3 \alpha-4 \beta, x+4 \alpha-$ $3 \beta+5 m \in D_{3}$ by applying (2). By a similar way, one can show that for any integer $j, x+2 j(\alpha-2 \beta), x+$ $2 i(\alpha-2 \beta)+\alpha \in D_{3}$ and $x+(2 i+1)(\alpha-2 \beta)+5 m, x+(2 i+1)(\alpha-2 \beta)+\alpha+5 m \in D_{3}$. Therefore for any integers $r$, s, both $x+r(2 \alpha+\beta)+s(\alpha-2 \beta)+5(r+s) m$ and $x+r(2 \alpha+\beta)+s(\alpha-2 \beta)+\alpha+5(r+s) m$ belong to $D_{3}$.

From now one, we assume that $0, a, 2 a+b+5 m \in D_{3}$ for our convenience. Then by Lemma 10(3), $r(2 a+b)+s(a-2 b) \in D_{3}$ if and only if $r+s$ is even. This implies that $(2 a+b)-(a-2 b)=a+3 b \in D_{3}$ and $(2 a+b)+(a-2 b)=3 a-b \in D_{3}$. Let $H$ be a subgroup of $\mathbb{Z}_{10 m}$ generated by $a+3 b$ and $3 a-b$, namely $H=\langle a+3 b, 3 a-b\rangle$. Now we have the following lemma.

Lemma 11. For a subgroup $H=\langle a+3 b, 3 a-b\rangle$ of $\mathbb{Z}_{10 m}$, we have

the set $H \cup(H+a)$ is a subset of $D_{3}$ and $H \cap(H+a)=\varnothing$, $\mathbb{Z}_{10 m}$ is generated by $a$ and $b$, and the index of $H$ in $\mathbb{Z}_{10 m}$ is exactly 10.

Proof of Lemma 11. (1) For any $x \in H$, there exist integers $i, j$ such that $x=i(a+3 b)+j(3 a-b)$. Since $a+3 b=(2 a+b)-(a-2 b)$ and $3 a-b=(2 a+b)+(a-2 b)$, we have $x=i(a+3 b)+j(3 a-b)=$ $(i+j)(2 a+b)+(j-i)(a-2 b)$. By Lemma 10(3), $x$ and $x+a$ belong to $D_{3}$, and hence $H \cup(H+a)$ is a subset of $D_{3}$.

Suppose that $y \in H \cap(H+a) \neq \varnothing$. Then $a \in H$, and hence $\langle a\rangle \subset H \subset D_{3}$. Now $a$ is dominated by 0 and $2 a$, a contradiction. Therefore $H \cap(H+a)=\varnothing$.

(2) Let $k$ be the order of $2 a+b$ in $\mathbb{Z}_{10 m}$. If $k$ is odd, then $k(2 a+b)+5 m=5 m \in D_{3}$ by Lemma 10(3). It contradicts the assumption that $D_{3}$ is a type-III efficient total dominating set of $G$. So $k$ is even, and let $k=2 k^{\prime}$. Now we have $k^{\prime}(2 a+b)=5 m$. So $\mathbb{Z}_{10 m}$ is generated by $a$ and $b$.

Let $t$ be the index of $H$ in $\mathbb{Z}_{10 m}$. Since $\left|D_{3}\right|=\frac{10 m}{5}=2 m$ and $|H \cup(H+a)|=2 \times \frac{10 m}{t}$, the index $t$ is at least 10 . 
For any $x \in \mathbb{Z}_{10 m}$, there exist integers $i, j$ such that $x=i a+j b$. Since $x=i a+j b=i(a+3 b)+(j-3 i) b$, $x \in H+(i-3 j) b$. This implies that any coset of $H$ can be expressed by $H+r b$ for some integer $r$. Because $10 b=3(a+3 b)-(3 a-b) \in H$, the index $t$ of $H$ is at most 10 . Therefore the index of $H$ in $\mathbb{Z}_{10 m}$ is exactly 10 .

Proof of Theorem 2(3). By a construction in Section 3, we know that if $\{ \pm a, \pm b\} \equiv\{1,3,7,9\}(\bmod 10)$, then $G=C_{10 m}[a, b, 5 m]$ has a type-III efficient total dominating set.

Conversely, assume that $G$ has a type-III efficient total dominating set $D_{3}$. By Lemma 8 , we may assume $0, a, 2 a+b+5 m \in D_{3}$. By Lemma 10(3), for any integers $r, s, x+r(2 a+b)+s(a-2 b)+5(r+$ s) $m, x+r(2 a+b)+s(a-2 b)+a+5(r+s) m \in D_{3}$.

Let $k$ be the order of $2 a+b$ in $\mathbb{Z}_{10 m}$. If $k$ is odd, then $k(2 a+b)+5 m=5 m \in D_{3}$ by Lemma 10. It contradicts the assumption that $D_{3}$ is a type-III efficient total dominating set of $G$. So $k$ is even, and let $k=2 k^{\prime}$. Now we have $k^{\prime}(2 a+b)=5 m$. If $k^{\prime}$ is even, $k^{\prime}(2 a+b)=5 m \in D_{3}$, which is a contradiction. Hence $k^{\prime}$ is odd.

Since $-(a-2 b)(2 a+b)+(2 a+b)(a-2 b)=0 \in D_{3}$, it holds that $-a+2 b+2 a+b=a+3 b$ is even by Lemma 10. It means that $a$ and $b$ have the same parity. If both $a$ and $b$ are even, then $5 m=k^{\prime}(2 a+b)$ is also even, which implies that all generators of $G$ are even. It contradicts that $G$ is connected. Therefore both $a$ and $b$ are odd. This means that $5 m=k^{\prime}(2 a+b)$ is also odd, which implies that $m$ is also odd.

By Lemma 11(2), for any $y \in H, y \equiv 0(\bmod 10)$. This implies that $a+3 b \equiv 0$ and $3 a-b \equiv 0(\bmod 10)$. Since both $a$ nd $b$ are odd, all possible pair $(a(\bmod 10), b(\bmod 10))$ are $(1,3),(3,9),(5,5),(7,1)$ and $(9,7)$. If $(a(\bmod 10), b(\bmod 10))$ is $(5,5)$, then all generators of $G$ are multiple of 5 , which contradicts that $G$ is connected. For all other cases, it holds that $\{ \pm a, \pm b\} \equiv\{1,3,7,9\}(\bmod 10)$. In this case, $H$ is $\langle 10\rangle$ and $H+a$ is $\langle 10\rangle+i$, where $i$ is $1,3,7$ or 9 depending on $(a(\bmod 10), b(\bmod 10))$.

Author Contributions: The contributions of all authors are equal. All authors have read and agreed to the published version of the manuscript.

Funding: The first and second authors were supported by the Basic Science Research Program through the National Research Foundation of Korea (NRF) funded by the Ministry of Education (2018R1D1A1B05048450) and (2020R1I1A3A04036669), respectively.

Conflicts of Interest: The authors declare no conflict of interest.

\section{References}

1. Haynes, T.W.; Hedetniemi, S.T.; Slater, P.J. Fundamentals of Domination in Graphs; Marcel Dekker: New York, NY, USA, 1998.

2. Sabidussi, G. On a class of fixed-point-free graphs. Proc. Amer. Math. Soc. 1958, 9, 800-804. [CrossRef]

3. Dejter, I.J.; Serra, O. Efficient dominating sets in Cayley graphs. Discrete Appl. Math. 2003, 129, 319-328. [CrossRef]

4. Reji Kumar, K.; MacGillivray, G. Efficient dominating sets in circulant graphs. Discret Math. 2013, 313, 767-771. [CrossRef]

5. Gavlas, H.; Schultz, K.; Slater, P. Efficient open domination in graphs. Sci. Ser. A Math. Sci. 2003, 6, 77-84.

6. $\quad$ Bakker, E.M.; Leeuwen, J.V. Some Domination Problems on Trees and General Graphs; Technical Report RUU-CS-91-22; Department of Information and Computing Sciences, Utrecht University: Utrecht, The Netherlands, 1991.

7. Schaudt, O. On weighted efficient total domination. J. Discret. Algorithms 2012, 10, 61-69. [CrossRef]

8. Lee, J. Independent perfect domination sets in Cayley graphs. J. Graph Theory 2001, 37, 213-219. [CrossRef]

9. MacGillivray, G.; Warren, J.M. Efficient total dominating sets in Cayley graphs. Util. Math. 2014, 94, 347-359.

10. Obradović, N; Peters, J.; Ružić, G. Efficient domination in circulant graphs with two chord lengths. Inf. Process. Lett. 2007, 102, 253-258. 
11. Kwon, Y.S.; Sohn, M.Y. Existence of efficient total domination sets of circulant graphs of degree 4. Discrete Math. 2020, 343, 111742. [CrossRef]

12. Feng, R.; Huang, H.; Zhou, S. Perfect codes in circulant graphs. Discrete Math. 2017, 340, 1522-1527. [CrossRef]

Publisher's Note: MDPI stays neutral with regard to jurisdictional claims in published maps and institutional affiliations.

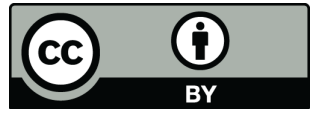

(C) 2020 by the authors. Licensee MDPI, Basel, Switzerland. This article is an open access article distributed under the terms and conditions of the Creative Commons Attribution (CC BY) license (http:/ / creativecommons.org/licenses/by/4.0/). 